\title{
Impact on Brain due to Alcoholism using Improved Fuzzy C-Regression based Alcohol Detection
}

\author{
Gayathriselvaraj, M.Punithavalli
}

\begin{abstract}
In general, two risk factors such as alcohol expectancy and impulsivity have been concerned with alcohol abuse Currently, many people have been addicted to alcoholism and have an Alcohol Use Disorder (AUD) that affects neurons behavior in the human brain. Still, how such risk factors interrelate to estimate the alcoholism. To solve this problem, Fuzzy C-Regression based Alcoholism Detection (FCRAD) method has been proposed that segments the Region-Of-Interests (ROIs) from the human brain image to predict the Gray Matter Volume (GMV) reduction in the right posterior insula in women and the left thalamus in both men and women efficiently. However, it requires the detection of GMV reduction in the other brain image regions. This multi-modality can decrease the fuzziness of the partition and the crisp membership degrees were not derived easily. Therefore in this article, the GMV reduction in other regions of the brain images including right posterior insula in women and left thalamus in both men and women has been detected, an Improved FCRAD (IFCRAD) method is proposed to simplify the segmentation of the brain images by considering the second regularization term in the objective function of the FCR to take into account the noisy data. Also, the Euclidean distance is replaced with the Voronoi distance for computing different fuzzy membership functions. Moreover, new error measure and reward function are used in the objective function of the FCR to reward nearly crisp membership functions and to obtain more crisp partition. So, the brain images are segmented into gray-matter images that derive the ROIs to analyze the GMV reduction with less complexity. Finally, the experimental results illustrate the proposed IFCRAD method achieves higher accuracy than the existing AD methods.
\end{abstract}

Keywords : Alcohol use disorder, Gray matter volume, Fuzzy $C$-regression, ROI segmentation, Voronoi diagram

\section{INTRODUCTION}

Alcohol dependency also known as alcoholism and it is a chronic syndrome represented by the inability to control the consumption or absorption level of alcohol.

Revised Manuscript Received on December 30, 2019.

* Correspondence Author

Gayathriselvaraj*, Department of Computer Applications, Bharathiar University , Coimbatore. Email: selvamgayathri06@gmail.com

Dr. M. Punithavalli, Department of Computer Applications, Bharathiar University , Coimbatore. Email: punithavalli@buc.edu.in

(C) The Authors. Published by Blue Eyes Intelligence Engineering and Sciences Publication (BEIESP). This is an open access article under the CC-BY-NC-ND license http://creativecommons.org/licenses/by-nc-nd/4.0/
Due to this inability, several indirect and direct effects are caused wherein the direct effects are riskier than the indirect effects. The direct effects are shortness of breath, shrink brain actions, blurry visualization, lowered anxiety and stress, etc., whereas the indirect effects are brain deficiencies, liver syndrome, heart diseases, Osteoporosis, genetic disorders, high possibility of cancer, etc [1]. To estimate the actual consumption level of alcohol, different trustworthy experiments are offered in which the most familiar experiment is Blood Alcohol Content (BAC) test used to validate alcohol tolerance. Nowadays, data mining algorithms have been developed to predict the most drastic damages of the human brain due to alcoholism. Since data mining algorithms deal with the difficulty of how better to utilize the data for obtaining new information and enhancing the decision-making process. Therefore, such algorithms are applied in medical sciences for providing a perfect diagnosis to the patient syndrome according to the most significant features from the large-scale datasets [2]. The extraction of those significant features is also achieved by different methods such as regression, clustering, association rule mining and classification. Over the previous years, structural brain imaging i.e., brain structure characterized with Magnetic Resonance Imaging (MRI) has been emerged to observe the correlation between the risk features and problem drinking [3]. The risk features include alcohol expectancy and impulsivity estimated by the General Linear Models (GLM). The alcohol expectancy may correlate with the decreased Gray Matter Volume (GVM) of the right posterior insula in women and the correlation of alcohol expectancy and impulsivity may relate to the decreased GMV of the left thalamus in both men and women. Moreover, the structural brain correlates of these risk variables were predicted by a Voxel-Based Morphometry (VBM) analysis. Nonetheless, this analysis was used only for predicting the linear distribution of GMV. To tackle this problem, Fuzzy C-Regression (FCR) model has been proposed that helps to predict the non-linearity distribution of GMV in the brain image [4]. In this model, the noise in the brain images was eliminated by adding the spatial information. Then, the Regions-Of-Interest (ROI) such as the right posterior insula in women and the left thalamus in men and women were segmented to detect the GMV by averaging the vocal GMV densities in each individual region. 


\section{Impact on Brain due to Alcoholism using Improved Fuzzy C-Regression based Alcohol Detection}

Moreover, the Alcohol Expectancy Test (AET) score was estimated based on the deviations of GMV that mediates the correlation between GMV and alcoholism. This AET score was mostly related to the reduced GMV of the left thalamus in women and men combined and in men alone. By using this model, the difficulty of segmenting and predicting GMV reduction in both insula and thalamus regions was reduced.

On the other hand, it needs to detect the GMV reduction in other brain image regions which may reduce the fuzziness of the partition and the determination of crisp membership degrees during segmentation. Hence in this article, initially the FCRAD is applied to segment different ROIs of brain images like cerebrum, cerebellum, hippocampus, etc., along with the right posterior insula in women and left thalamus in both men and women. Further, to increase the fuzziness of the partition and determine the crisp membership degrees, an Improved FCRAD (IFCRAD) method is proposed that consider the second regularization term in the objective function in order to allow for the noisy data. As well, Voronoi distance is used instead of Euclidean distance to compute different fuzzy membership functions for different ROIs. Besides, new error measure i.e., Voronoi distance and reward function are used in the objective function of the FCR to reward nearly crisp membership functions and obtain a more crisp partition. Thus, the ROIs of brain images are segmented to analyze the GMV reduction and the correlation between AET score and alcoholism.

The remaining part of this paper is organized as Section II reviews the related researches on segmenting and classifying the MRI images of human brain. Section III explains the methodology of proposed algorithm. Section IV analyzes the efficiency of proposed algorithm and compares with the existing algorithms. Section V concludes the entire research work.

\section{LITERATURE SURVEY}

Zhang et al. [5] proposed a Neural Network (NN)-based method for classifying the given MRI brain images as normal or abnormal. Initially, wavelet transform was employed for extracting the features from images and the Principal Component Analysis (PCA) was used for reducing the features dimensions. Then, the reduced features were fed to the BPNN in which Scaled Conjugate Gradient (SCG) was adopted to discover the optimal weights of the NN. However, the feature extraction phase was most time-consuming.

Jafari \& Kasaei [6] proposed a NN-based method for automatic classification of MRI of brain under three classes such as normal, lesion benign and malignant. This method has six phases: preprocessing, seeded region growing segmentation, Connected Component Labeling (CCL), feature extraction, feature dimension reduction and classification. In the preprocessing phase, the enhancement and restoration techniques were used for providing a more suitable image for the subsequent processes. The seeded region growing segmentation was used for splitting the image into significant regions. Then, each pixel was labeled and the features were extracted using the Discrete Wavelet Transform (DWT). After that, the dimension of the extracted DWT features was reduced by using the PCA for obtaining more significant features. At last, a supervised feed-forward Back-Propagation (BP) NN was used for classifying the subjects to normal or abnormal. However, it requires new training for each time whenever there was an increase in image database.Nanthagopal \& Sukanesh [7] designed a computer software system for segmentation and classification of brain computed tomography images. Initially, 2D DWT was used to eliminate the noise from the images. Then, the features were extracted and the most optimal features were chosen by using Student's t-test. Further, the selected features were fed to the Support Vector Machine (SVM) and Probabilistic NN (PNN) classifiers to classify the normal and abnormal brain images. However, it requires new training for the classifiers whenever there was a change in the dataset. Kalbkhani et al. [8] proposed a robust algorithm for detecting the type of diseases in brain MRI. Initially, two-level 2D WT of input image was computed and the wavelet coefficients of detail sub-bands were modeled by Generalized Autoregressive Conditional Heteroscedasticity (GARCH) statistical model. The parameters of this model were considered as the primary feature vector. Once the feature vectors were normalized, PCA and LDA were used for extracting the appropriate features and eliminating the redundancy from the primary feature vector. At last, the extracted features were applied to the K-Nearest Neighbor (KNN) and SVM for classifying the normal image or disease type. However, the classification algorithms consume more time for training. Sahu et al. [9] proposed a new approach for classifying normal and abnormal brain MRI by using Bi-dimensional Empirical Mode Decomposition (BEMD) and Autoregressive (AR) model. Initially, brain MRI was decomposed into four Intrinsic Mode Functions (IMFs) by using BFMD and AR coefficients from multiple IMFs were concatenated for constructing a feature vector. Then, a binary classifier such as Least-Squares SVM (LS-SVM) was applied to classify the brain MRI as normal or abnormal. However, the kernel parameters used in this method were chosen based on the trial and error method. It requires an automatic selection of kernel parameters and kernel function to increase the classification accuracy while a large database was used.

Wang et al. [10] proposed a deep Convolutional NN (CNN) for segmenting the MRI brain images. In this method, three networks such as network of $2 x$ upsampled prediction, network of $4 x$ upsampled prediction and network of $8 x$ upsampled prediction were constructed. The datasets was obtained from IBSR database of Neuroimaging Informatics Tools and Resources Clearinghouse (NITRC). However, these datasets were not very effective since it has only T1 images. For T1 images, this method was not effective. Zhao et al. [11] proposed a supervised brain tumor segmentation method which identifies the dissimilar tumoral structures of both high grade and low grade gliomas in MRI based on two types of features, namely the gradient features and the context-sensitive features. The gradient variation was captured by using both 2D and 3D gradient information. Moreover, a circular context-sensitive feature was proposed to capture the context information. 
The features were compressed and optimized based on an mRMR algorithm. Also, the Random forest classifier was used for classifying voxels based on the compact feature set. However, this method has less sensitivity, f-score and positive predictive value.

\section{PROPOSED METHODOLOGY}

In this section, the proposed IFCRAD method for detecting the GMV reduction in different regions of brain images is described brief. In this proposed technique, the brain images of both men and women are given to FCR model to segment different ROIs of brain images such as cerebrum, cerebellum, cerebellar vermis, caudate, putamen, hippocampus, thalamus, basal ganglia, globus pallidus and amygdale along with the right posterior insula in women and the left thalamus in both men and women brain images. Conversely, this causes reduction in fuzziness of the partition and determination of more crisp fuzzy membership degrees to segment the ROIs. As a result, an IFCRAD method is proposed to segment the ROIs of brain images as follows.

The main difference between FCRAD and proposed IFCRAD method is replacing Euclidean distance with the Voronoi distance and modifying the objective function of FCR by using reward function and error measure to obtain more crisp partition corresponding to the local linear model. Normally, a Takagi-Sugeno (TS) model has a set of fuzzy rules, each explaining a local input-output correlation as: $R_{c}:$ IF $\dot{i}_{k 1}$ is $A_{1}^{c}$ and $\ldots$ and $\dot{i}_{k M}$ is $A_{M}^{c}$, THEN $y_{c}=b_{c}^{0}+b_{c}^{1} i_{k 1}+\cdots+b_{c}^{M} i_{k M}, c=1, \ldots, r$

In Eq. (1), $R_{c}$ is the $c^{\text {th }}$ IF-THEN rule, $A_{j}^{c}$ denotes the fuzzy subset and $r$ is the number of rules. Consider the set of input-output sample image pairs is

$S=\left\{\left(i_{1}, o_{1}\right),\left(i_{2}, o_{2}\right), \ldots,\left(i_{N}, o_{N}\right)\right\}=$ $\left\{\left(i_{k}, o_{k}\right)\right\}, k=1, \ldots, N$

where $N$ denotes the total number of samples, $i_{k}=\left[i_{k 1}, i_{k 2}, \ldots, i_{k M}\right] \in \mathbb{R}^{M}$ refers to the input image vector, $M$ is the number of fuzzy models, $y_{k} \in \mathbb{R}$ refers to the output and $\theta_{c}=\left[b_{c}^{0}, b_{c}^{1}, \ldots, b_{c}^{M}\right] \in \mathbb{R}^{M+1}{ }_{,} c=1, \ldots, r$ is the parameter vector of the corresponding local linear model. Let the data pairs in $S$ are drawn from $C$ different clusters. The hyper-plane of the $c^{\text {th }}$ cluster is represented as:

$$
\begin{gathered}
y_{k}=f_{c}\left(i_{k}, \theta_{c}\right)+E_{c k}\left(\theta_{c}\right) \\
=b_{c}^{0}+b_{c}^{1} i_{k 1}+\cdots+b_{c}^{M} i_{k M}+E_{c k}\left(\theta_{c}\right) \\
y_{k}=\left[x_{k}, 1\right] \cdot \theta_{c}^{T}+E_{c k}\left(\theta_{c}\right), c=1, \ldots, r
\end{gathered}
$$

Typically, FCR model utilizes polynomials as cluster prototypes. By using real functions $\mathbb{R} \rightarrow \mathbb{R}$, the cluster prototypes are characterized by the coefficients of the polynomial i.e., the prototypes are factors of $\mathbb{R}^{q+1}$ where $q$ denotes the degree of the polynomials. The Euclidean distance $E_{c k}\left(\theta_{c}\right)$ of FCM is replaced by the residual error $|y-h(i)|$ of a data sample $(i, y)$ to the polynomial $h$.
To simplify, consider the extended data samples $\hat{\imath}$ which have an additional factor $\hat{\iota}_{0} \equiv 1$. After that, the distance function can be rewritten as follows:

$$
E_{c k}^{2}\left(\theta_{c}\right)=\left(y_{k}-\theta_{i}^{T} \hat{\imath}_{k}\right)^{2}
$$

The coefficients $\theta_{c}$ are also obtained in similar manner as cluster centers of FCM as Eq. (2), only the prototype update can be replaced according to the modified distance function as:

$\theta_{c}=\left(\sum_{k=1}^{N} \mu_{c k}^{m}\left(\hat{\iota}_{k} \hat{l}_{k}^{T}\right)\right)^{-1}\left(\sum_{k=1}^{N} \mu_{c k}^{m} y_{k} \hat{\iota}_{k}\right)$

Consider a crisp partition which is encouraged by the cluster centroids. Normally, the resulting partition is referred to as the Voronoi diagram. The Euclidean distance of a data sample $\vec{i}_{k}$ to the hyperplane that splits the clusters $p_{c}$ and $p_{s}$ is given by $\left|\left(i_{k}-h_{s}\right)^{T} \mathcal{N}_{s}\right|$ where $h_{s}$ denotes the pixel location on the hyperplane i.e., $h_{s}=p_{s}+p_{c} / 2$ and $\mathcal{N}_{s}$ denotes the normal vector $\mathcal{N}_{s}=\beta_{s} \cdot\left(p_{c}-p_{s}\right)$

$$
\text { with } \beta_{s}=1 /\left\|p_{c}-p_{s}\right\| \text { for } s \neq c \text {. By considering }
$$

the absolute value of the minimum over all the directed distances yields the distance to the edge of the cell. If $\vec{i}_{k}$ lies within the Voronoi cell of cluster $k$, then the distance to the cell is zero. This special case is formalized by setting $\beta_{s}=1$ and defining as:

$$
E_{c k_{V}}\left(\theta_{c}\right)=\left|\min _{1 \leq s \leq r}\left(i-h_{s}\right)^{T_{n_{s}}}\right|
$$

To obtain the crisp partition for different ROIs, noise is taken as an individual class. It is represented by a fictitious model that has a Voronoi distance $\left(E_{c k_{V}}\left(\theta_{c}\right)\right)$ from all the pixels. The membership $\mu_{* k}$ of pixel $\dot{t}_{k}$ in the noise cluster is given by,

$$
\mu_{* k}=1-\sum_{c=1}^{r} \mu_{c k}
$$

Thus, the membership constraint for the best clusters is effectively decreased to

$$
\sum_{c=1}^{r} \mu_{c k}<1
$$

The new objective function is as follows:

$$
\begin{aligned}
& I(S ; U, \theta)=\sum_{k=1}^{N} \sum_{c=1}^{r}\left(\mu_{c k}^{m}\right) E_{c k}^{2}\left(\theta_{c}\right)+ \\
& \sum_{k=1}^{N} E_{c k}^{2}\left(\theta_{c}\right) \mu_{* k}^{m}-\sum_{k=1}^{N} a_{l} \sum_{l=1}^{r}\left(\mu_{l k}-\frac{1}{2}\right)^{2}
\end{aligned}
$$

In Eq. (8), the first term on the right hand side is similar as the actual FCR objective function whereas the second term is due to the noise clustering. Likewise, the third term is the reward function. If the pixel $x_{k}$ is assigned to one prototype $p_{c}$, then for all other $c \neq l, \mu_{c k}=1$ and $\mu_{l k}=0$ For all these cases, the third term evaluates to $-a_{l} / 4$ If the membership degrees become more fuzziness, the third term increases. Since it is found that Eq. (8) needs to minimize and this modification rewards crisp membership degrees.

Published By: 


\section{Impact on Brain due to Alcoholism using Improved Fuzzy C-Regression based Alcohol Detection}

The constrained problem $/$ with respect to $\mu_{c k}$ is solved by introducing $N$ Lagrange multipliers $\lambda_{k}, k=1, \ldots, N$ The minimization of $I$ is initiated by forming the Lagrangian as:

$$
\mathcal{L}=J-\sum_{k=1}^{N} \lambda_{k}\left(\sum_{c=1}^{r} \mu_{c k}+\mu_{* k}-1\right)
$$

By differentiating the Lagrangian with respect to $\mu_{c k}, \mu_{* k}$ and $\lambda_{k}$ also setting the derivatives to zero, obtain:

$$
\begin{aligned}
& \frac{\partial \mathcal{L}}{\partial \mu_{c k}}=m \mu_{c k}^{m-1} E_{c k}^{2}-\lambda_{k}=0 \\
& \frac{\partial \mathcal{L}}{\partial \mu_{* k}}=m E_{c k_{V}}^{2} \mu_{* k}^{m-1}-\lambda_{k}=0 \\
& \frac{\partial \mathcal{L}}{\partial \lambda_{k}}=\sum_{c=1}^{r} \mu_{c k}+\mu_{* k}-1=0
\end{aligned}
$$

From Eqns. (10) and (11), obtain:

$$
\begin{aligned}
& \mu_{c k}=\left[\frac{\lambda_{k}}{m}\right]^{\frac{1}{m-1}}\left[\frac{1}{E_{c k}^{2}}\right]^{\frac{1}{m-1}} \\
& \mu_{* k}=\left[\frac{\lambda_{k}}{m}\right]^{\frac{1}{m-1}}\left[\frac{1}{E_{c k}^{2}}\right]^{\frac{1}{m-1}}
\end{aligned}
$$

By using Eqns. (12)-(14), obtain:

$$
\left[\frac{\lambda_{k}}{m}\right]^{\frac{1}{m-1}}=\frac{1}{\sum_{l=1}^{r}\left(\frac{1}{E_{l k}}\right)^{\frac{2}{m-1}}+\left(\frac{1}{E_{l k V}}\right)^{\frac{2}{m-1}}}
$$

After that, the following equation can be obtained by substituting Eq. (15) into Eq. (13):

$$
\mu_{c k}=\frac{1}{\sum_{l=1}^{r}\left(\frac{E_{c k}}{E_{l k}}\right)^{\frac{2}{m-1}}+\left(\frac{E_{c k V}}{E_{l k V}}\right)^{\frac{2}{m-1}}}, c \neq l
$$

From Eqns. (2) and (8), the objective function of the novel FCR clustering algorithm is defined as follows:

$$
\begin{aligned}
& I(S ; U, \theta)=\sum_{k=1}^{N} \sum_{c=1}^{r}\left(\mu_{c k}^{m}\right)\left(y_{k}-\theta_{i}^{T} \hat{\imath}_{k}\right)^{2}+ \\
& \sum_{k=1}^{N}\left|\min _{1 \leq s \leq r}\left(i-h_{s}\right)^{T} n_{s}\right|\left(1-\sum_{c=1}^{r} \mu_{c k}\right)^{m} \\
& =\sum_{k=1}^{N} \sum_{c=1}^{r}\left(\mu_{c k}^{m}\right)\left(y_{k}-\sum_{j=1}^{M+1} \theta_{i j} \hat{l}_{k j}\right)^{2}+ \\
& \sum_{k=1}^{N}\left|\min _{1 \leq s \leq r}\left(i-h_{s}\right)^{T} n_{s}\right|\left(1-\sum_{c=1}^{r} \mu_{c k}\right)^{m}
\end{aligned}
$$

The partial derivative of the objective function in Eq. (17) is as:

$$
\begin{aligned}
& \frac{\partial j}{\partial \theta_{i j}}=-2 \sum_{k=1}^{N} \mu_{c k}^{m}\left(y_{k}-\sum_{j=1}^{M+1} \theta_{i j} \hat{\imath}_{k j}\right) \hat{\imath}_{k j}(\mathbf{1 8}) \\
& \text { Then, } \\
& \theta_{i j}=\frac{\sum_{k=1}^{N} \mu_{c k}^{m}\left(y_{k}-\sum_{t \neq j} \theta_{i t} \hat{\imath}_{k t}\right) \hat{\imath}_{k j}}{\sum_{k=1}^{N} \mu_{c k}^{m} \hat{\imath}_{k j}^{2}}, c=1, \ldots, r_{j} j= \\
& 1_{, \ldots,}, M+1
\end{aligned}
$$

As a result, the fuzzy membership degrees for different ROIs are obtained and the misclassified pixels from noisy

regions are avoided. This iteration process is repeated until the maximum error measure i.e., distance between two clusters at two successive iterations is less than a termination threshold. After the convergence, defuzzification is applied to assign each pixel to a particular cluster for which the membership degree is maximal. Therefore, this clustering algorithm is used to segment different ROIs from the entire brain image of both men and women.

\section{Algorithm: IFCRAD}

Input: Brain image dataset of both men and women

Output: Detected GMV reduction at different parts of brain images

Given a training image, set $m>1$ and the parameter vectors $\theta_{i}$ at random;

Choose the termination threshold $\epsilon>0$ and initialize $U^{(0)}$ randomly;

\section{for(each training image)}

\{

Choose the number of clusters at random; Initialize the fuzziness factor;

$$
\operatorname{for}(t=1,2, \ldots, \infty)
$$

\{

Calculate the error measure $E_{c k}^{2}\left(\theta_{c}\right)$ by using Eq. (3);

Calculate the new error measure $E_{c k_{V}}\left(\theta_{c}\right)$ via Eq. (5);

Compute $\mu_{c k}^{(t)}$ and $\theta_{i j}^{(t)}$ via Eqns. (16) and (19) respectively;

Update $U^{(t)}$ with $E_{c k}\left(\theta_{c}^{(t)}\right)$ and $E_{c k_{V}}\left(\theta_{c}^{(t)}\right)$ to satisfy,

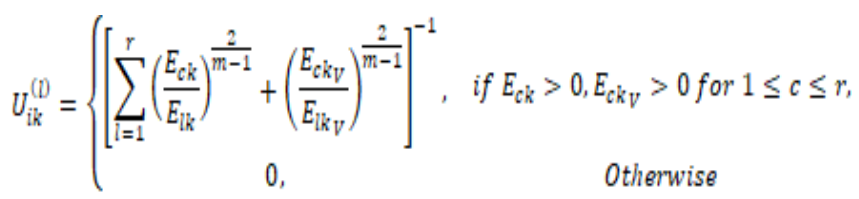

$$
\text { if }\left(e r r=\left\|U^{(t)}-U^{(t-1)}\right\| \leq \epsilon\right)
$$

\{

Compute the fuzzy cost function via Eq. (17);

else

$$
\begin{aligned}
& \qquad t=t+1 ; \\
& \text { end } \text { if } \\
& \text { end } \text { for } \\
& \text { Segment ROIs; } \\
& \text { Find GMV reduction at different regions in brain images of } \\
& \text { both men and women; } \\
& \text { Observe the alcohol expectancy level; } \\
& \text { end for } \\
& \text { end }
\end{aligned}
$$

\section{end}




\section{RESULTS AND DISCUSSION}

In this section, the performance of the proposed IFCRAD method is evaluated and compared with the existing FCRAD [4] and GLMAD [3] methods by using MATLAB 2018a.

This experiment is conducted by using MIRIAD dataset that consists of 780 T1-weighted images in which 188 are men with alcoholic condition, 116 are men with the healthy condition, 238 are women with an alcoholic condition and
138 are women with the healthy condition. Among those brain images, a total of 400 images from each category are used in this analysis. Here, 180 images are taken for training and the rest of 220 images are considered for testing. The comparison analysis is performed in terms of precision, recall, f-measure and accuracy.

The segmented image results of brain images under different conditions for existing and proposed methods are shown in Table 1.

Table.1 Outcomes of Segmented Brain Images(Gray Matter)under Different Conditions

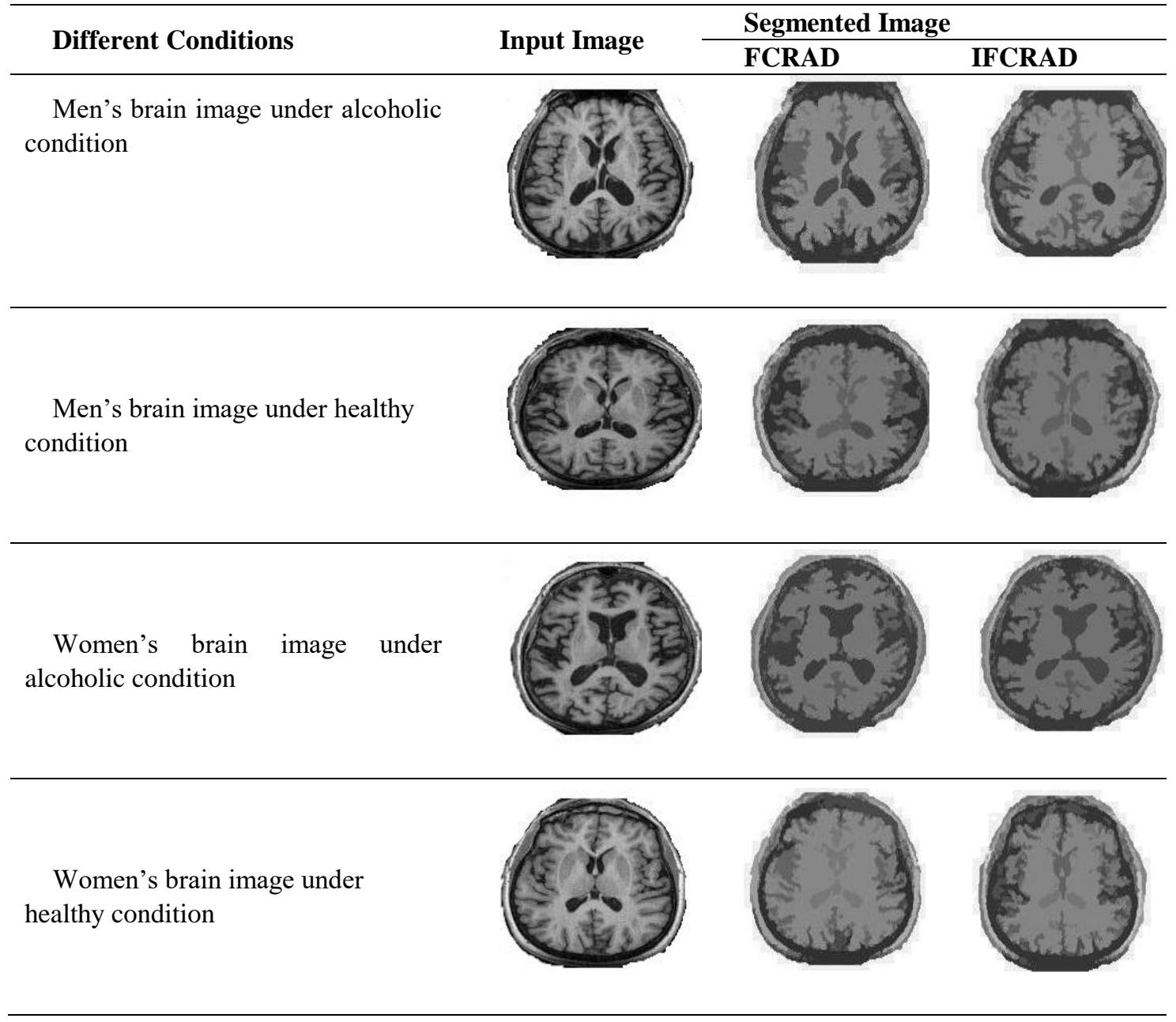

Table 2 and 3 show the outcomes of the detected GMV reduction at different regions of brain images for FCRAD and IFCRAD methods using both women and men under an alcoholic and healthy condition, respectively. 
Impact on Brain due to Alcoholism using Improved Fuzzy C-Regression based Alcohol Detection

Table.2 Outcomes of Detected GMV Reduction at Different Regions of Women's Brain Images under both Alcoholic \& Healthy Conditions

\begin{tabular}{|c|c|c|c|c|}
\hline \multirow{2}{*}{$\begin{array}{l}\text { Different } \\
\text { ROIs }\end{array}$} & \multicolumn{2}{|c|}{$\begin{array}{c}\text { Women's Brain Images under Alcoholic } \\
\text { Condition }\end{array}$} & \multicolumn{2}{|c|}{$\begin{array}{c}\text { Women's Brain Images under Healthy } \\
\text { Condition }\end{array}$} \\
\hline & FCRAD & IFCRAD & FCRAD & IFCRAD \\
\hline \multicolumn{5}{|c|}{ Cerebrum } \\
\hline \multicolumn{5}{|c|}{ Cerebellum } \\
\hline \multicolumn{5}{|c|}{ Amgdata } \\
\hline \multicolumn{5}{|c|}{ Basal Neuclei } \\
\hline \multicolumn{5}{|c|}{ Putamen } \\
\hline \multicolumn{5}{|c|}{ Thalamus } \\
\hline \multicolumn{5}{|c|}{ Hippocampus } \\
\hline \multicolumn{5}{|c|}{ Cerebellar Vermis } \\
\hline Caudate & & & & \\
\hline
\end{tabular}

Retrieval Number: A5309119119/2019@BEIESP DOI: 10.35940/ijitee.A53091.129219

Journal Website: www.ijitee.org
Published By:

Blue Eyes Intelligence Engineering \& Sciences Publication

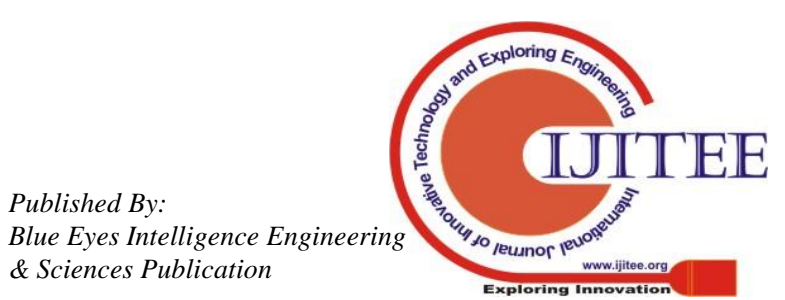


Globus Pallidus
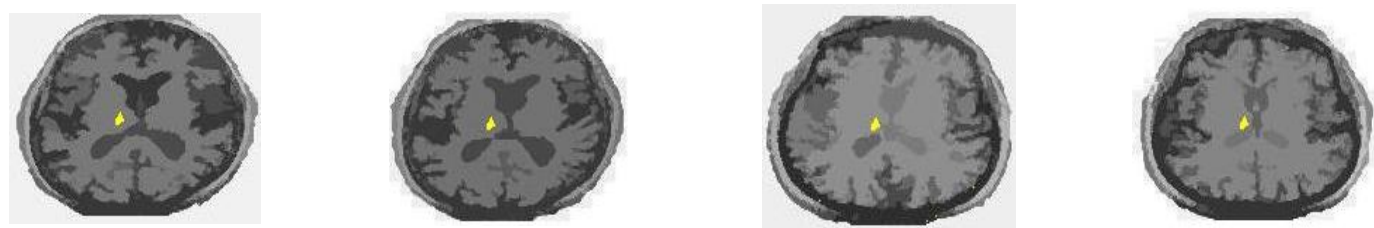

Table.3 Outcomes of Detected GMV Reduction at Different Regions of Men's Brain Images under both Alcoholic \& Healthy Conditions

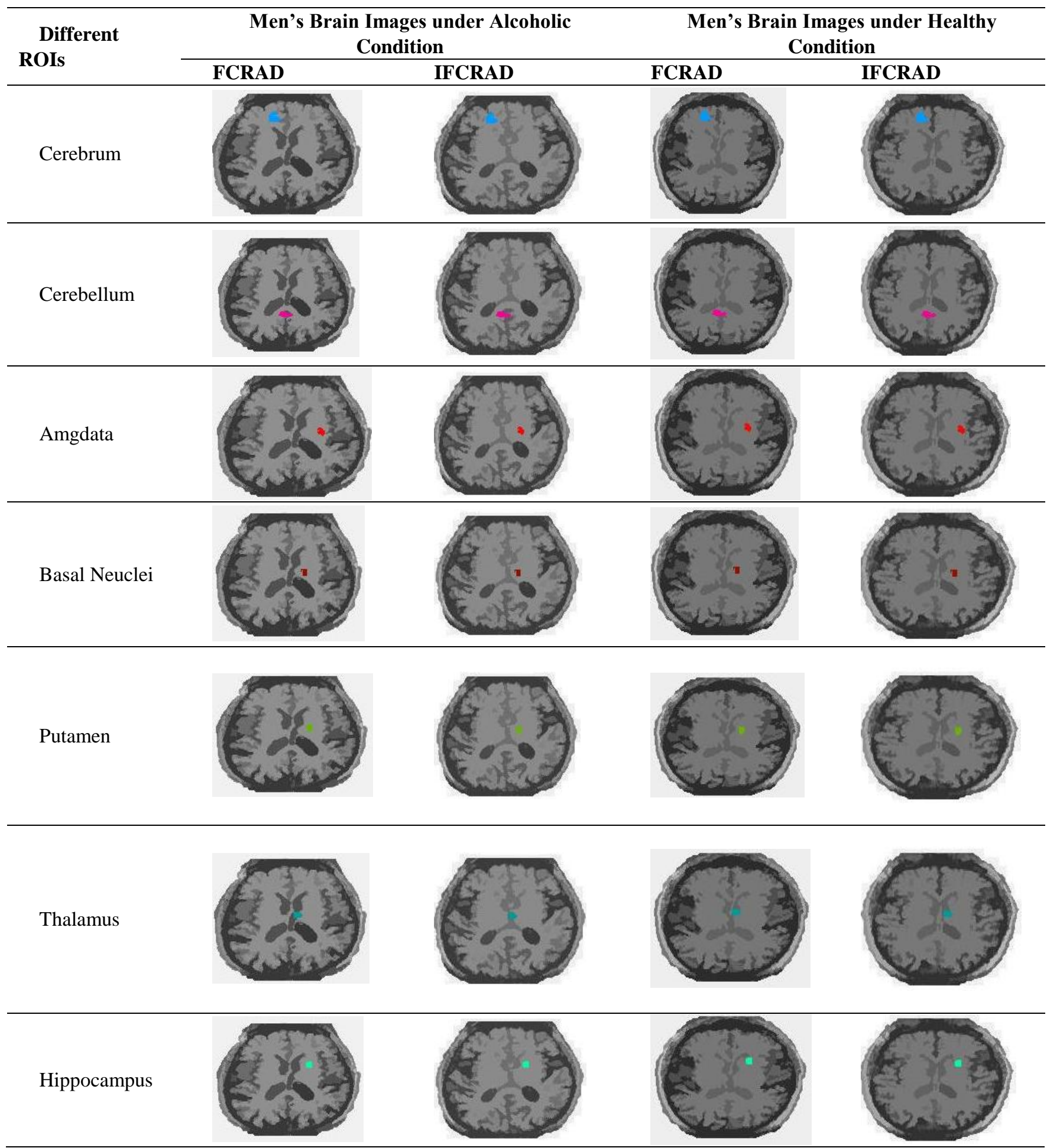

Retrieval Number: A5309119119/2019@BEIESP DOI: 10.35940/ijitee.A53091.129219

Journal Website: www.ijitee.org
Published By:

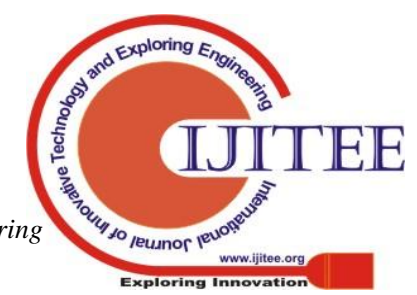


Cerebellar

Vermis
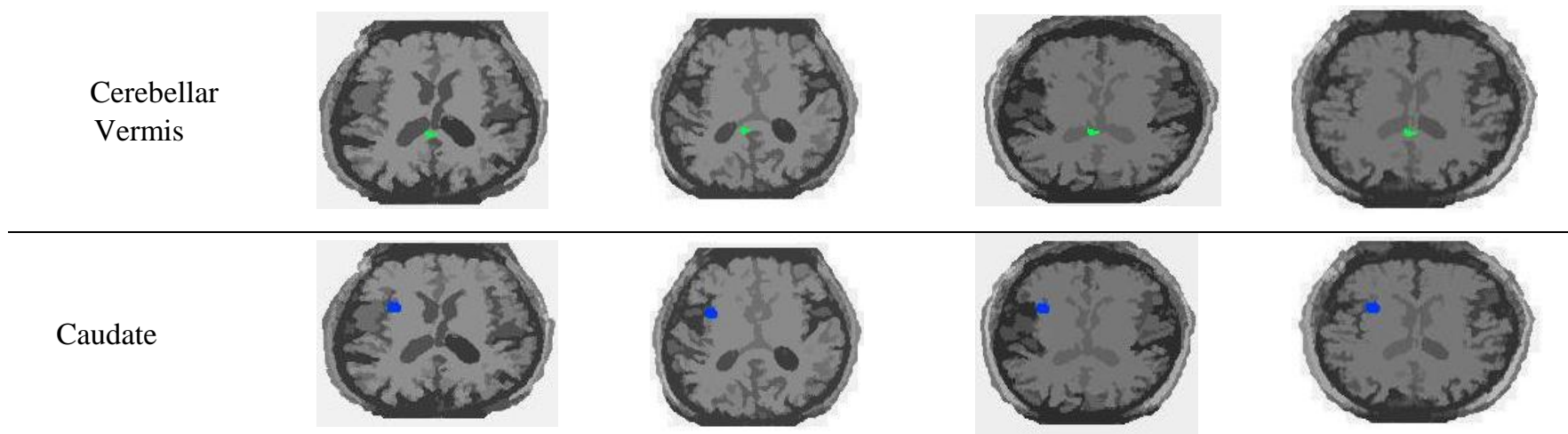

Globus Pallidus
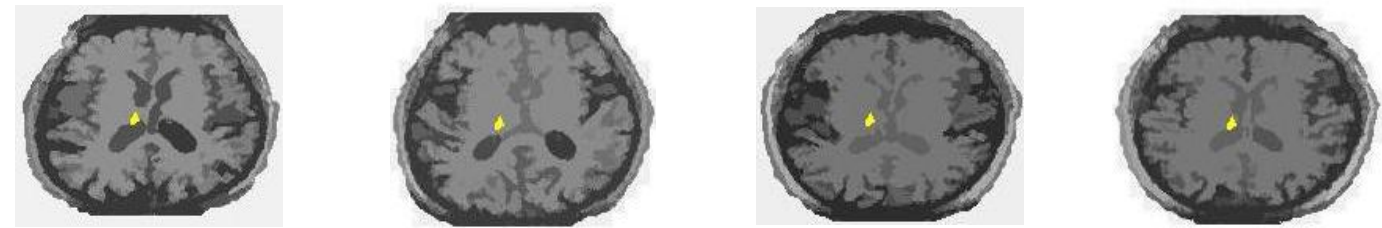

The mean \pm SD GMV for training images using FCRAD and IFCRAD methods are given in Table 4.

Table.4 Mean \pm SD GMV for Brain Images using IFCRAD Method

\begin{tabular}{|l|c|c|c|c|}
\hline $\begin{array}{c}\text { No. of } \\
\text { Images }\end{array}$ & $\begin{array}{c}\text { Men with Alcohol } \\
\text { Condition }\end{array}$ & $\begin{array}{c}\text { Men with Healthy } \\
\text { Condition }\end{array}$ & $\begin{array}{c}\text { Women with Alcohol } \\
\text { Condition }\end{array}$ & $\begin{array}{c}\text { Women with Healthy } \\
\text { Condition }\end{array}$ \\
\hline 50 & $363.78 \pm 51.66$ & $326.90 \pm 59.03$ & $368.13 \pm 66.25$ & $316.57 \pm 65.81$ \\
\hline 100 & $363.70 \pm 51.39$ & $326.62 \pm 59.02$ & $368.91 \pm 66.04$ & $316.46 \pm 65.75$ \\
\hline 150 & $363.63 \pm 51.02$ & $326.47 \pm 58.99$ & $368.79 \pm 65.89$ & $316.35 \pm 65.68$ \\
\hline 200 & $363.56 \pm 49.86$ & $326.21 \pm 58.96$ & $368.56 \pm 65.68$ & $316.24 \pm 65.59$ \\
\hline 250 & $363.44 \pm 49.63$ & $326.08 \pm 58.94$ & $368.33 \pm 65.56$ & $316.13 \pm 65.50$ \\
\hline
\end{tabular}

\section{A. Precision}

It is calculated based on the AD at True Positive (TP) and False Positive (FP) rates.

$$
\text { Precision }=\frac{T P}{T P+F P}
$$

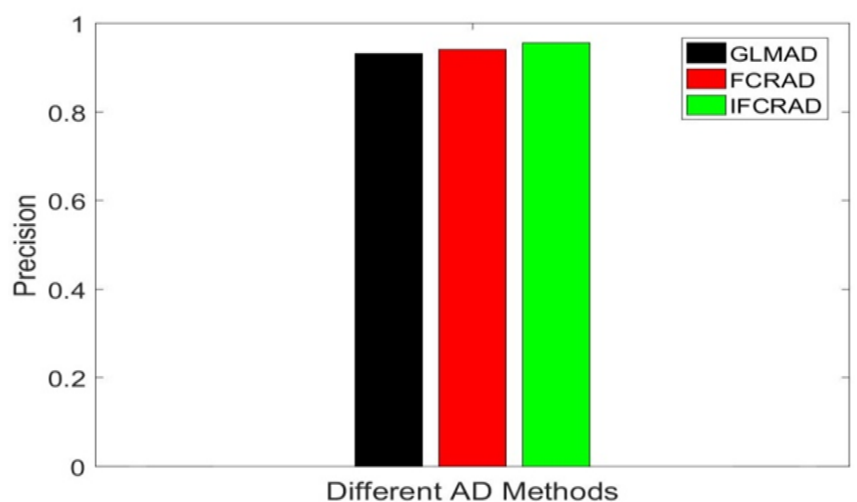

Figure.1 "Comparison of Precision

Figure 1 shows the precision of IFCRAD, FCRAD and GLMAD methods. Through this analysis, it is identified that the precision of the IFCRAD method is $2.69 \%$ higher than the GLMAD method and 1.59\% higher than the FCRAD method.

\section{B. Recall}

It is calculated based on the AD at TP and False Negative (FN) rates.

$$
\text { Recall }=\frac{T P}{T P+F N}
$$

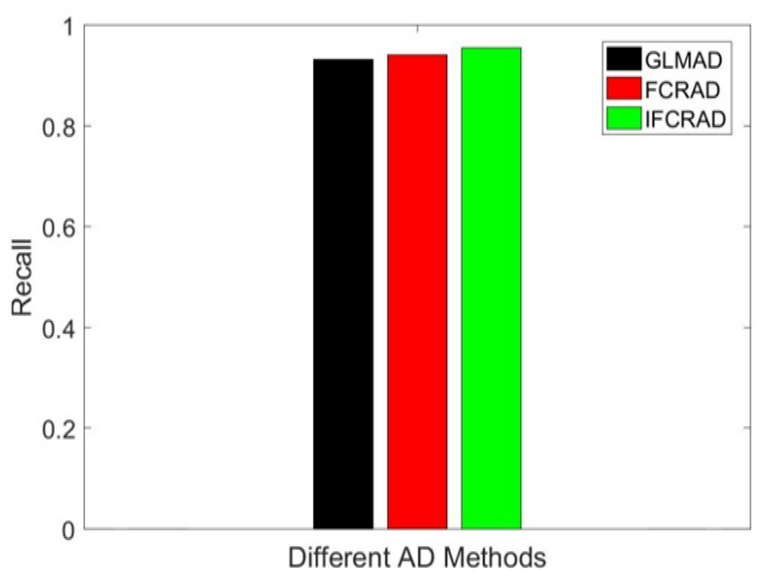

Figure.2 Comparison of Recall

Figure 2 shows the recall of IFCRAD, FCRAD and GLMAD methods. Through this analysis, it is identified that the recall of the IFCRAD method is $2.47 \%$ higher than the GLMAD method and $1.38 \%$ higher than the FCRAD method.

\section{F-Measure}

It is calculated by using both precision and recall as:

$$
F-\text { measure }=2 \times\left(\frac{\text { Precision } \times \text { Recall }}{\text { Precision }+ \text { Recall }}\right)
$$




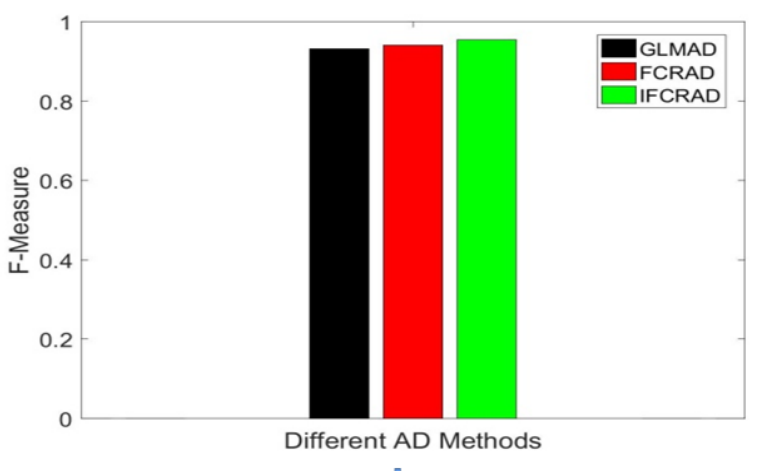

Figure.3 Comparison of F-measure

Figure 3 shows the f-measure of IFCRAD, FCRAD and GLMAD methods. Through this analysis, it is identified that the f-measure of the IFCRAD method is $2.58 \%$ higher than the GLMAD method and $1.48 \%$ higher than the FCRAD method

\section{Accuracy}

It is defined as the fraction of both TP and True Negatives (TN) among the total number of cases analyzed.

$$
\text { Accuracy }=\frac{T P+T N}{T P+T N+F P+F N}
$$

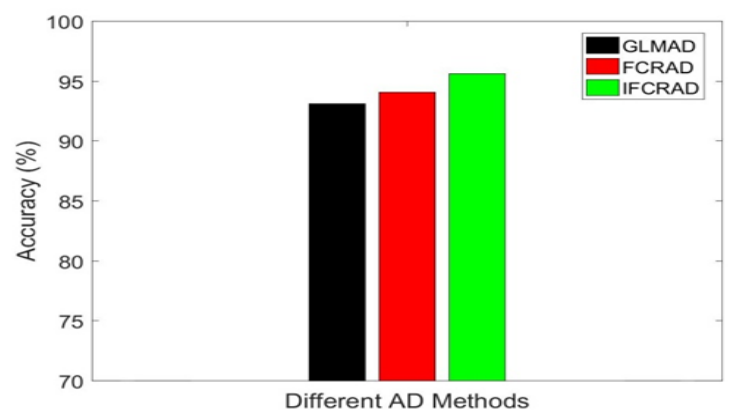

Figure 4 shows the accuracy of IFCRAD, FCRAD and GLMAD methods. Through this analysis, it is identified that the accuracy of the IFCRAD method is $2.7 \%$ higher than the GLMAD method and $1.67 \%$ higher than the FCRAD method.

\section{CONCLUSION}

In this article, an IFCRAD method is proposed to detect the alcoholism level by segmenting different ROIs from the brain images such as cerebrum, cerebellum, hippocampus, etc., including the right posterior insula in women and left thalamus in both men and women. In this method, the fuzziness of the partition is improved by considering the new error measure based on Voronoi diagram and reward function that can determine the crisp fuzzy membership degrees for obtaining more crisp ROIs of the brain images. After that, GMV value reduction at each segmented region is analyzed to estimate the AET score that can be used to predict the alcoholic effects in both men and women efficiently. Finally, the experimental results proved that the proposed IFCRAD method achieves an accuracy of $95.63 \%$ which is $2.18 \%$ higher than all other existing $\mathrm{AD}$ methods. Likewise, the precision, recall and f-measure values of IFCRAD method are approximately $2 \%$ higher than all existing methods. As a result, it is concluded that the proposed IFCRAD method can detect the GMV reduction at different regions of the brain images along with the alcoholic effects in both men and women efficiently

\section{REFERENCES}

1. Enoch, M. A., \& Goldman, D. (2002). Problem drinking and alcoholism: diagnosis and treatment. American family physician, 65(3), 441-454.

2. Mathew, S. T., \& Nachamai, M. (2015). Clustering of brain MRI image using data mining algorithm. International Journal of Advanced Computational Engineering and Networking, 3(4), 37-40.

3. Ide, J. S., Zhornitsky, S., Hu, S., Zhang, S., Krystal, J. H., \& Chiang-shan, R. L. (2017). Sex differences in the interacting roles of impulsivity and positive alcohol expectancy in problem drinking: A structural brain imaging study. Neuroimage: clinical, 14, 750-759.

4. Gayathri, S., Punithavalli, M., \& Chaitanya, V. K. (2019) Alcoholexpectancy prediction using fuzzy c-regression based structural brain imaging. International Journal of Intelligent Engineering and Systems, 12(5), 139-148.

5. Zhang, Y., Dong, Z., Wu, L., \& Wang, S. (2011). A hybrid method for MRI brain image classification. Expert Systems with Applications, 38(8), 10049-10053.

6. Jafari, M., \& Kasaei, S. (2011). Automatic brain tissue detection in MRI images using seeded region growing segmentation and neural network classification. Australian Journal of Basic and Applied Sciences, 5(8), 1066-1079.

7. Nanthagopal, A. P., \& Sukanesh, R. (2013). Wavelet statistical texture features-based segmentation and classification of brain computed tomography images. IET image processing, 7(1), 25-32.

8. Kalbkhani, H., Shayesteh, M. G., \& Zali-Vargahan, B. (2013). Robust algorithm for brain magnetic resonance image (MRI) classification based on GARCH variances series. Biomedical Signal Processing and Control, 8(6), 909-919.

9. Sahu, O., Anand, V., Kanhangad, V., \& Pachori, R. B. (2015) Classification of magnetic resonance brain images using bi-dimensiona empirical mode decomposition and autoregressive model. Biomedical Engineering Letters, 5(4), 311-320.

10. Wang, Y., Sun, Z., Liu, C., Peng, W., \& Zhang, J. (2016, August). MRI image segmentation by fully convolutional networks. In 2016 IEEE International Conference on Mechatronics and Automation (pp. 1697-1702). IEEE.

11. Zhao, J., Meng, Z., Wei, L., Sun, C., Zou, Q., \& Su, R. (2019). Supervised brain tumor segmentation based on gradient and context-sensitive features. Frontiers in Neuroscience, 13.

\section{AUTHORS PROFILE}

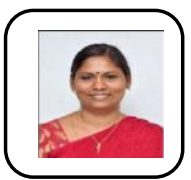

Dr .M. Punithavalli is working as an Professor in the Department of Computer Applications at Bharathiar University, Coimbatore. She is Doctorate in computer Science having 21 years of Research experience. She has published more than 80 research paper Articles in International Journals. She has authored 3books

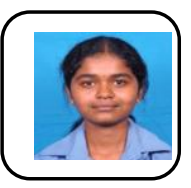

Gayathriselvaraj pursuing Ph.D at Bharathiar University, Coimbatore. M.Phil from Mother Teresa University, Kodaikanal and Postgraduate from Bannari Amman Institute of Technology, Sathyamangalam 